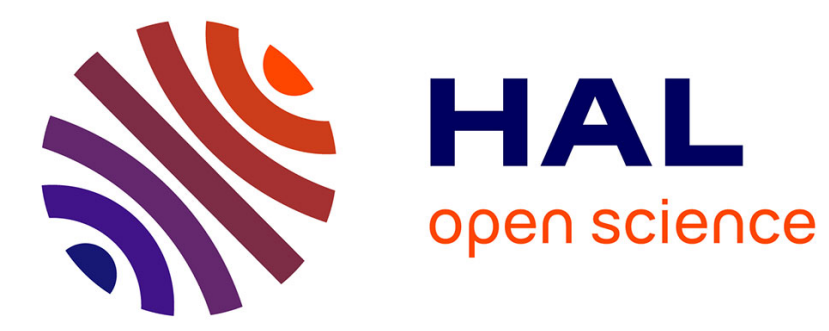

\title{
Recent data on adipose tissue, insulin resistance, diabetes and dyslipidaemia in antiretroviral therapy controlled HIV-infected persons
}

Jacqueline Capeau, Claire Lagathu, Véronique Béreziat, Bruno Fève

\section{- To cite this version:}

Jacqueline Capeau, Claire Lagathu, Véronique Béreziat, Bruno Fève. Recent data on adipose tissue, insulin resistance, diabetes and dyslipidaemia in antiretroviral therapy controlled HIV-infected persons. Current Opinion in HIV and AIDS, 2021, Publish Ahead of Print, 10.1097/COH.0000000000000674 . hal-03187463

\section{HAL Id: hal-03187463 \\ https: / hal.sorbonne-universite.fr/hal-03187463}

Submitted on 1 Apr 2021

HAL is a multi-disciplinary open access archive for the deposit and dissemination of scientific research documents, whether they are published or not. The documents may come from teaching and research institutions in France or abroad, or from public or private research centers.
L'archive ouverte pluridisciplinaire HAL, est destinée au dépôt et à la diffusion de documents scientifiques de niveau recherche, publiés ou non, émanant des établissements d'enseignement et de recherche français ou étrangers, des laboratoires publics ou privés. 
Recent data on adipose tissue, insulin resistance, diabetes and dyslipidemia in ARTcontrolled HIV-infected persons

Jacqueline Capeau ${ }^{1}$, Claire Lagathu ${ }^{1}$, Véronique Béréziat $^{1}$, Bruno Fève $^{1,2}$

${ }^{1}$ Sorbonne Université-Inserm, Faculty of Medicine, Centre de Recherche Saint-Antoine (CRSA), UMR_S938, ICAN, Paris, France

${ }^{2}$ Department of Endocrinology, CRMR Prisis, Saint-Antoine Hospital, GH APHP-Sorbonne Université, Paris France

Corresponding author: Jacqueline Capeau

Faculty of Medicine Sorbonne Université

27 rue Chaligny 75012 Paris, France

Phone: 33668456988

Email: jacqueline.capeau@inserm.fr 
Structured abstract

Purpose of review: Increased total body fat with truncal redistribution is common in antiretroviral-treatment(ART)-controlled persons living with-HIV(PLWH), leading to insulin resistance, prediabetes/diabetes and dyslipidemia. We address these topics here.

Recent findings: Most antiretrovirals are associated with gain in trunk fat, including visceral adipose tissue(VAT). Protease-inhibitors(PI) could inhibit white fat ability to dissipate energy (i.e. beiging) favoring fat gain. Expansion of VAT is associated with a pro-inflammatory profile linked to the tryptophan-kynurenine pathway and CD4 subtypes. ART-associated increased AT quantity leads to decreased AT density, insulin resistance and dyslipidemia that could be improved by lifestyle modifications.

PLWH present high level of insulin resistance, regardless of their treatment, and a higher prevalence of prediabetes, but not diabetes, than non-infected persons. Otherwise, HbA1c values appear inaccurate to diagnose prediabetes/diabetes in PLWH.

ART-related-dyslipidemia is characterized by elevated LDL-C and/or high triglycerides and reduced HDL-C. Whereas treatment with PIs generally results in worsened lipid values, treatment with integrase-strand-transfer-inhibitors is associated with a better profile. Tenofovir-alafenamide is associated with higher lipid levels than tenofovir-disoproxilfumarate. Treatment of LDL-C-dyslipidemia could benefit, in statin-insufficiently controlled patients, from the class of proprotein-convertase-subtilsin-kenin-type-9(PCSK-9) inhibitors.

Summary: Lifestyle modifications are mandatory to reduce fat and improve dysglycemia/dyslipidemia. New drugs can efficiently control diabetes and LDL-C-dyslipidemia

Keywords

Adipose tissue, insulin resistance, diabetes, dyslipidemia, antiretroviral 


\section{1- Introduction}

Antiretroviral-treatment (ART)-controlled persons living with HIV (PLWH) often present with central fat redistribution, characterized by increased visceral (VAT) and reduced subcutaneous adipose tissue (SAT). VAT expansion is associated with ectopic fat deposition in muscle, heart, liver, and pancreas, resulting in cardio-metabolic comorbidities(1).The prevalence of obesity is increasing worldwide, as a result of increased sedentary lifestyle and harmful food habits. This is also a concern for PLWH, even if they generally exhibit a lower prevalence of obesity than the corresponding general population.

Continuous ART, required to control HIV, results in potential ART-related outcomes altering AT and lipids. HIV, hidden within immune cells in fat reservoirs, releases viral proteins that can affect fat, and could act in synergy with some antiretrovirals(2).

Fat expansion and related cardio-metabolic outcomes are frequent in PLWH, mainly in relation to age and personal or environmental factors. Currently used antiretrovirals have less impact on glucose and lipid levels than first generation antiretrovirals (except fat gain induced by some integrase strand transfer inhibitors (INSTIs) and tenofovir alafenamide (TAF), see the paper by J Lake and J Trevillyan in this issue).

\section{2- Current fat alterations in PLWH}

The initial recognition of fat alterations in PLWH on ART began in the mid 1990s with highly prevalent facial and limb lipoatrophy, ultimately found to be a side effect of thymidine analogue reverse transcriptase inhibitors (tNRTIs). These drugs were replaced in the early 2000s in Western countries (and later in low-medium income countries) by ART with less effect on fat and lipoatrophy regressed in most lipoatrophic patients. 


\section{2-1 Epidemiology}

Importantly, tNRTI-related lipoatrophy is still present in some PLWH, with increased waistto-hip ratio (WHR) reflecting increased waist (WC) and decreased hip circumferences (HC). These long-lasting alterations were associated, in the COCOMO cohort, with low AT density and impaired adiponectin production(3) and also with increased total cholesterol (TC) and low HDL-C(4). Increased WHR and/or lipoatrophy, reminiscent of stavudine use, are also associated with insulin resistance in long-term infected and treated PLWH from the APROCO cohort(5) and with incident diabetes among PLWH from the MACS cohort(6).

Over time, PLWH are gaining weight both in the truncal and limb compartments as shown in the European Modena cohort(7). In this cohort, the post-menopausal state was associated with greater fat mass. Peripheral lipoatrophy was diagnosed in one third of participants during the 2004-2014 period, more frequently among men. In addition, a CD4 nadir <200 cells $/ \mathrm{mm}^{3}$ was associated with greater trunk fat mass on the long-term. This latter finding could possibly be explained by a high level of fat reservoirs, enhancing the effect of viral proteins on $\mathrm{AT}(2)$.

In a representative US population of PLWH evaluated during the 2009-2012 period (Medical Monitoring Project, MMP), 50-64 year old women were more likely to be obese than men (8.4\% prevalence difference)(8). In the MACS and WIHS cohorts, including HIV-infected and non-infected men (MACS) and women (WIHS) with similar risk factors, BMI, HC and WC were greater in women than men and also higher in non-infected participants than in PLWH, both in men and women. However, the changes in these anthropometric variables did not differ according to the HIV status(9). BMI increased until age 60 and then plateaued, whereas WC and WHR continued to increase irrespective of HIV serostatus, indicating age-related truncal 
fat redistribution. Moreover, in HIV-infected women, the rate of fat gain did not increase during/after menopause in contrast to HIV-negative women who experienced increased BMI and $\mathrm{WC}(10)$.

The impact of currently used PIs, NRTIs and non-NRTIs (NNRTIs) on fat gain appears mild. Currently used PIs are associated with modest fat gain: boosted atazanavir (ATV/r) with truncal fat and boosted darunavir (DRV/r) with increased VAT and SAT(11,12). Efavirenz (EFV) has not been previously associated with fat alterations. However, results from the ADVANCE trial suggest that EFV impaired weight gain among PLWH bearing the CYP2B6 genotypes leading to slow and intermediate EFV metabolism, and therefore to higher EFV concentrations(13). Tenofovir disoproxil fumarate (TDF) is also proposed to prevent fat gain. The role of INSTIs and TAF on fat gain is discussed in another paper in the present issue.

\section{2-2 Mechanisms}

Several lines of evidence suggest that the virus itself is probably involved into AT modifications. AT is an HIV/SIV (simian immunodeficiency virus) reservoir and SIV/HIV infection has been associated with features of adipose tissue aging. Thus, fat from SIVinfected macaques displayed markers of senescence. HIV proteins Tat and Nef released by infected immune cells could exert a bystander effect on adipose cells, enhancing senescence and impairing adipocyte differentiation and function(14).

PIs themselves can modify AT. Recently, inhibition of fat beiging, a process allowing dissipation of excessive fat through heat production, has been proposed to explain fat gain. The expression of the micro-RNA processing enzyme DICER and of the canonical brown and beige genes (UCP-1, PGC1alpha, PRDM16) were reduced in SAT of PLWH and negatively correlated with initial HIV infection severity and duration of PI use $(11,15,16)$. These findings 
suggest a PI-induced shift from a brown/beige to a white fat phenotype. As a result, fat is no longer dissipating energy as heat but rather retaining lipids and evolving toward hypertrophy and possible fat gain. This mechanism could also result from initial HIV infection severity, leading to increased fat reservoirs and AT dysfunction.

Decreased total and trunk fat was associated, in postmenopausal women from WIHS, with increases in the bone-derived hormone, undercarboxylated-osteocalcin, an indicator of bone health that mediates glucose metabolism and regulates fat mass(17). This draws attention to potential links between bone and AT metabolism in PLWH that need to be further explored. Fat density, measured during CT scan imaging of VAT and SAT at L4-L5 level, can assess fat quality, in addition to quantity. It is proposed that, in the case of unhealthy expansion (hypertrophy), adipocytes become lipid engorged, of poorer quality and less dense. Fat density decreased over 96 weeks after ART initiation with ATV/r, DRV/r or raltegravir (RAL), each given with TDF/emtricitabine(FTC) as background. Coupled with increased SAT and VAT quantity, this suggests that unhealthy adipocyte hypertrophy occurs following ART initiation regardless of the molecule(18).

\section{2-3 Consequences}

VAT expansion is associated with ectopic fat deposition in liver, muscle, heart (epicardial fat), vessels and the pancreas, with deleterious cardiometabolic outcomes $(1,11)$. Increased abdominal fat, as evaluated by WHR, was associated with pro-inflammatory products of the tryptophan-kynurenine pathway, which could be a potential source of inflammation in HIV(19). Increased VAT was also related to reduced PBMC telomere length in PLWH, independently of smoking and age, suggesting involvement of VAT in accentuated aging(20). Body fat parameters were associated with hypogonadism in HIV-infected men <50y, 
inversely related to free testosterone and the estrone+estradiol/testosterone ratio, and were also associated with multimorbidity and frailty. Therefore, body fat mass, multimorbidity and frailty are associated with each other and contribute to male hypogonadism in PLWH(21).

Decreased SAT and VAT density, in favor of unhealthy fat hypertrophy, has been associated with increased HOMA-IR and triglyceride (TG) levels and decreased HDL-C, thus linking unhealthy AT to insulin resistance and dyslipidemia(22). Moreover, SAT of diabetic and nondiabetic PLWH was found to be enriched in CD4 and CD8 T effector memory (TEM) subsets compared to those present in blood, which could contribute to local tissue inflammation. The level of CD4/CD8 TEM subsets was even higher in SAT from diabetic as compared to non-diabetic PLWH(23). In diabetic subjects, SAT was enriched in a population of CD4 T cells expressing CX3CR1, GPR56 and CD57 which could exert anti-viral activities locally against HIV, CMV and other viruses (24).

In addition, in well-controlled PLWH, higher amounts of VAT was associated with higher atherosclerotic coronary plaque and coronary artery calcium score (CAC) while the reverse was seen with increased SAT(25), linking VAT and cardiovascular outcomes.

Apart from cardiometabolic comorbidities, altered AT distribution is associated with neurocognitive outcomes. In the MACS cohort, increased BMI and higher WC were associated with lower cognition and greater cognitive decline both in PLWH and in HIVnegative men(26).

\section{2-4 Interventions}

Lifestyle modifications are strongly encouraged: exercise, regardless of its intensity, resulted in reduced total and visceral AT in PLWH(27). 


\section{3- Insulin resistance, prediabetes and diabetes}

The high occurrence of diabetes noted with first-generation ART mostly resolved with newer ART(28). However, since some INSTIs and TAF can increase BMI, this could increase the risk of prediabetes/diabetes.

\section{3-1 Insulin resistance}

In routine clinical practice, insulin sensitivity and identification of insulin resistance is usually accomplished by calculating the HOMA-IR index, using fasting glucose and insulin levels. While this is far less accurate than an insulin clamp, HOMA-IR is often elevated in PLWH $(>2)$, and is associated with increased truncal fat. This finding raises concerns about the possible evolution towards diabetes.

In ViiV healthcare trials(29), HOMA-IR was evaluated in PLWH initiated with dolutegravirincluding therapies. In the SPRING-1 trial of ART-naïve PLWH initiated with DTG vs EFV, 80\% of PLWH had HOMA-IR>2 at baseline, with further increase during follow-up. In the switch SWORD and STRIIVING trials, baseline HOMA-IR was $>2$ in $65-75 \%$ with a mild increase in the 2 arms over time. Even if 33\% of the subjects were overweight and $17 \%$ obese, this high level of initial HOMA in ART-naïve and ART-controlled PLWH is worrying. In the TANGO study, patients were switched to dolutegravir/lamivudine (DTG/3TC) or remained under a TAF-containing regimen (elvitegravir/cobicistat, EVG/c, in most cases). Again, baseline HOMA-IR was elevated (median 2.8 and 2.6, respectively) and mildly decreased or increased during follow-up(30). HOMA-IR is generally lower in European cohorts with lower BMI. Thus, in the ETRAL study, evaluating aging PI-treated PLWH switched to RAL/etravirine, median

BMI was $<25 \mathrm{~kg} / \mathrm{m}^{2}$ but median HOMA-IR was 1.7 in men, 1.9 in women, and increased in 
relation with weight gain in men and peri/post-menopausal women(31). High values of HOMA-IR reported in most studies, at least in Western countries, could be related to ART, but probably less so than an elevated BMI, trunk fat accumulation and age.

\section{3-2 Diagnosis of prediabetes and diabetes.}

To diagnose prediabetes/diabetes recent recommendations advise criteria based on glycated hemoglobin $(\mathrm{HbA} 1 \mathrm{c})$ values, respectively $5.7 \%<\mathrm{HbA} 1 \mathrm{c}<6.4 \%(39-47 \mathrm{mmol} / \mathrm{mol})$ for prediabetes and $\mathrm{HbA} 1 \mathrm{c}>6.5 \%$ ( $>48 \mathrm{mmol} / \mathrm{mol}$ ) for frank diabetes In the CICADA study from Tanzania, the prevalence of diabetes was $13 \%$ using $\mathrm{HbA} 1 \mathrm{c}$ and $6 \%$ using oral glucose tolerance test (OGTT) with only partial overlap among participants identified by the two tests(32). In Thailand, authors diagnosed dysglycemia using fasting glucose, $2 \mathrm{~h}$ post-prandial glucose and HbA1c levels: the agreement between the three estimations was weak and conversely $\mathrm{HbA1c}$ underestimated prediabetes prevalence (33). In African PLWH, HbA1c also underestimated the prevalence of diabetes(34), in particular in those with sickle cell disease(35). These studies underline the inadequacy of using $\mathrm{HbA} 1 \mathrm{c}$ to diagnose prediabetes/diabetes in several populations, inasmuch as this parameter can vary with hemoglobin variants, presence of hemolysis, red globular volume and treatment with some NRTIS.

\section{3-3 Prevalence of prediabetes and diabetes}

At present, the prevalence of diabetes is generally not different between PLWH and controls, but the BMI of PLWH is generally lower. In a study performed in Finland in 2017(36), the prevalence of diabetes was 13.2\% in PLWH, a figure not different from $14.5 \%$ in the general population but with a lower prevalence of obesity: $18.2 \%$ vs $23.9 \%$. 
Importantly, fasting glucose was higher in PLWH but HbA1c lower, further stressing the concerns about using $\mathrm{HbA1c}$ to diagnose prediabetes/diabetes in PLWH. Of note, the risk of dysglycemia for a given BMI was higher in PLWH than in the general population. In the MMP group of US PLWH, the prevalence of diabetes was higher by $13 \%$ in women than men $>65 y(8)$. In the MACS cohort, the risk for incident diabetes was evaluated in pre-diabetic subjects between 1999 and 2019. PLWH had a 40\% higher risk of becoming diabetic than non-infected individuals, even if they were younger and with a lower BMI. Longer use of stavudine and resulting increased WHR could explain this increased risk(6). In PLWH from the Asia-Pacific region, with a median age of 35 years, diabetes incidence was quite elevated at 10.8/1000 persons-year-of-follow-up and associated with later years of follow-up 20142017 as compared to 2011-2013, in addition to classical risk factors (age, sex, BMI)(37).

\section{3-4 Mechanisms}

In the APROCO cohort, $11 \%$ of long-term infected and ART-controlled PLWH, median age 49 years, were diabetic. Diabetes was associated independently with increased inflammation and oxidative stress(5). In PLWH from the MACS, history of AIDS was linked to incident diabetes, which may reflect continued immune system dysregulation and inflammation as drivers for diabetes(6).

In the WIHS cohort, women experienced increased levels of HbA1c only if they gained $\geq 5 \%$ weight on INSTI(38). Up to now there is no indication of an increased risk of diabetes, over that related to weight gain, in INSTI-treated PLWH.

In the MACS and the WIHS cohorts, plasma lipidomic profiles revealed that eleven lipid species were associated with the risk of diabetes. Most of them were elevated in ARTtreated PLWH, which suggests a specific link between lipid metabolism and HIV and the 
possibility that ART-related plasma lipidomic alterations may precede the development of diabetes(39).

\section{3-5 Consequences}

In the MACS cohort, abnormal glucose metabolism was associated with declines in gait speed and grip strength, regardless of HIV serostatus. Improved glucose control is an intervenable target to prevent progression of physical function limitation and the occurrence of frailty(40).

In 2936 US veterans with HIV, 628 had depression. They had a $24 \%$ increased risk of diabetes, and more severe depression was associated with higher risk of developing diabetes(41).

\section{3-6 Treatment}

Even if not tested in dedicated clinical trials, PLWH can benefit, in addition to the conventional antidiabetics (metformin and sulfonylurea), from GLP-1 (glucagon-like peptide1) receptor agonists, and SGLT2 (sodium-glucose co-transporter-2) inhibitors that, in addition to decreasing glycemia, also decrease weight and cardiovascular risk(1), and also preserve kidney function in the case of SGLT2 inhibitors. The antidiabetic class of DPP4 inhibitors has an anti-inflammatory effect on adipose tissue(11).

\section{4- Dyslipidemia}

ART-induced alterations in lipid levels are common in PLWH. In addition to ART, dyslipidemia results from personal factors such as age, familial history, sex, diet, and alcohol intake. Increased LDL-C or triglycerides (TG) and decreased HDL-C, are related to different metabolic 
situations. LDL-related dyslipidemia is independent from increased TG and decreased HDL-C as evaluated by the TG:HDL-C ratio $(\mathrm{mg} / \mathrm{dl})>3.5$ for men and 2.5 for women, a type of dyslipidemia often seen in diabetic patients and associated with insulin resistance(28).

\section{4-1 Prevalence}

Recent studies report the prevalence of dyslipidemia in different groups of PLWH and corresponding general population. In a Finish population, elevated TG:HDL-C was found in $27 \%$ of PLWH as compared to $18 \%$ in the general population, in agreement with the higher prevalence of dysglycemia(36). In the French APROCO cohort, LDL-related dyslipidemia was found in $28 \%$ and high TG:HDL-C in $9 \%$ of PLWH(5). In the CARMA-ENDO study, including HIV-infected and non-infected women from British Columbia, a higher proportion of PLWH (63\%) than HIV-negative (40\%) had dyslipidemia, mainly low HDL-C or high TG, despite a young median age (43.5 years)(42). In the US MMP group, women $>50 y$ were more likely to have high TC and LDL-C than men: respectively 9.9 and 5.1\% for age 50-64y and 18.8 and $15.6 \%$ for age $\geq 65 y(8)$.

\section{4-2 Mechanisms}

In the APROCO cohort, LDL-related dyslipidemia was associated with oxidative stress, while elevated TG:HDL-C ratio was associated independently with inflammation and oxidative stress(5). In the MACS cohort, lower levels of the atheroprotective small HDL-particles were associated with greater systemic inflammation. This may be a potential mechanism contributing to increased cardiovascular risk among PLWH(43). These results enlighten the potential role of inflammation/oxidative stress as drivers for dyslipidemia, in addition to ART and individual factors. 
INSTI use has been consistently associated with globally improved lipid parameters in switch situations (decreased LDL-C and TG, often improved HDL-C). However, PLWH were often switched off PI to initiate INSTI. The beneficial effect could result from stopping the PI and/or INSTI initiation. In the TANGO study, PLWH switched to DTG/3TC (8\% initially PItreated) were compared to PLWH taking TAF (mainly EVG/c/TAF). Over 48 weeks, DTG/3TC treatment decreased TC, LDL-C and TG levels as compared to the TAF arm(30). The difference between the two arms could result from the INSTI, cobicistat or TAF use.

In the WIHS, comparing women switched or not to an INSTI, HDL-C level was decreased only in those INSTI-treated and gaining $\geq 5 \%$ weight, stressing weight-related rather than INSTIrelated changes(38).

The different effect on lipids of TAF vs TDF, previously identified in clinical trials, was confirmed in the real life setting in several studies: switching TDF to TAF increased TC, LDL-C, HDL-C and TG while the LDL-C/HDL-C ratio remained stable $(44,45)$. When TAF was switched back to TDF, lipid levels returned to previous values(46).

\section{4-3 Treatment}

Statins remain the first option to decrease LDL-C. If insufficiently decreased, ezetimibe is an efficient and safe second-line option. However, in PLWH insufficiently controlled or intolerant to statins, evolocumab, a proprotein convertase subtilsin-kenin type-9(PCSK9) inhibitor, was well tolerated and further reduced LDL-C by $60 \%$ at $24 \mathrm{w}$ versus placebo. It also reduced other atherogenic lipid levels(47). It can be considered for lowering atherogenic lipoproteins in PLWH with high cardiovascular risk. 
A lipidomic profiling of 211 lipid species in 937 patients, HIV-infected or not, from the MACS and WIHS cohorts revealed that 12 species were associated with risk of carotid artery plaque and conventional blood lipids, most of them being elevated in ART-controlled PLWH suggesting a link both with HIV infection and ART(48).

\section{5- Conclusion}

Fat alterations and fat gain observed in PLWH are often associated with insulin resistance and dysglycemia, raising concerns for the possible latter occurrence of diabetes. Recent ART combinations are involved into metabolic disturbances, mainly due to their weight gain effect, with mild direct effects on glycemic and lipid parameters. Strategies to control AT and metabolic alterations favor lifestyle modifications to reduce weight. Efficient anti-diabetic and hypolipidemic drugs are now available to lower glycemia and LDL-C to reasonable targets.

\section{6- Acknowledgements}

None assistance was required for the redaction of this manuscript.

Financial support and sponsorship: support was obtained by RHU CARMMA grant (ANR-15RHUS-0003) and the Fondation pour la Recherche Médicale (EQU201903007868).

Conflict of interest relative to the work: none

\section{7- Bullets}

- Aging long-term ART-controlled persons-living with HIV often present fat redistribution with truncal fat inflation.

- Increased ART-related adipose tissue quantity is associated with unhealthy decreased density, resulting in insulin resistance and dyslipidemia. 
- Insulin resistance is common in PLWH, whatever antiretrovirals, and could result in prediabetes, which incidence is increased as compared to non-infected controls.

- HbA1c values are often used to diagnose prediabetes/diabetes but appear inadequate in PLWH, being lower than expected according to glycemia.

- PI use is associated with worsened lipid values (increased LDL-C and triglycerides, often reduced HDL-C) while integrase-strand-transfer inhibitors appear more lipid friendly.

\section{8- References}

1. Piche ME, Tchernof A, Despres JP. Obesity Phenotypes, Diabetes, and Cardiovascular Diseases. Circ Res. 2020;126(11):1477-500.

2. Koethe JR, Lagathu C, Lake JE, Domingo P, Calmy A, Falutz J, et al. HIV and antiretroviral therapy-related fat alterations. Nat Rev Dis Primers. 2020;6(1):48.

${ }^{* *} \mathrm{~A}$ general review on the different fat alterations observed in PLWH, the mechanisms involved and also on their management

3. Gelpi M, Knudsen AD, Larsen KB, Mocroft A, Lebech AM, Lindegaard B, et al. Longlasting alterations in adipose tissue density and adiponectin production in people living with HIV after thymidine analogues exposure. BMC Infect Dis. 2019;19(1):708.

4. Gelpi M, Afzal S, Fuchs A, Lundgren J, Knudsen AD, Drivsholm N, et al. Prior exposure to thymidine analogues and didanosine is associated with long-lasting alterations in adipose tissue distribution and cardiovascular risk factors. AIDS. 2019;33(4):675-83.

* An interesting report linking tNRTI-remanent abdominal fat alterations to dyslipidemia 
5. Bastard JP, Couffignal C, Fellahi S, Bard JM, Mentre F, Salmon D, et al. Diabetes and dyslipidaemia are associated with oxidative stress independently of inflammation in longterm antiretroviral-treated HIV-infected patients. Diabetes Metab. 2019;45(6):573-81.

6. Slama L, Barrett BW, Abraham A, Palella F, Kingsley L, Vlard JP, et al. Risk for Incident Diabetes is Greater in Pre-Diabetic Men with HIV than Without HIV. AIDS. 2021;in press.

* An interesting study linking stavudine-remanent increased WHR to prediabetes and showing an increased risk of diabetes in prediabetic PLWH

7. Debroy $\mathrm{P}, \mathrm{Sim} \mathrm{M}$, Erlandson KM, Falutz J, Prado CM, Brown TT, et al. Progressive increases in fat mass occur in adults living with HIV on antiretroviral therapy, but patterns differ by sex and anatomic depot. J Antimicrob Chemother. 2019;74(4):1028-34.

** An important study evaluating the evolution of fat mass in PLWH on the long-term.

8. Frazier EL, Sutton MY, Tie Y, Fagan J, Fanfair RN. Differences by Sex in Cardiovascular Comorbid Conditions Among Older Adults (Aged 50-64 or $>/=65$ Years) Receiving Care for Human Immunodeficiency Virus. Clin Infect Dis. 2019;69(12):2091-100.

9. Erlandson KM, Lake JE. Fat Matters: Understanding the Role of Adipose Tissue in Health in HIV Infection. Curr HIV/AIDS Rep. 2016;13(1):20-30.

10. Nguyen TTJ, Ma Y, Scherzer R, Adimora AA, Fischl M, French AL, et al., editors. Body composition changes over the menopausal transition in women with and at risk for HIV. CROI 2020 \#658; 2020; Boston.

11. Godfrey C, Bremer A, Alba D, Apovian C, Koethe JR, Koliwad S, et al. Obesity and Fat Metabolism in Human Immunodeficiency Virus-Infected Individuals: Immunopathogenic Mechanisms and Clinical Implications. J Infect Dis. 2019;220(3):420-31.

** A general review presenting some of the novel mechanisms related to fat alterations and their clinical consequences 
12. Adrian S, Miao H, Feng H, Scherzinger A, Nardini G, Beghetto B, et al. Effects of atazanavir, darunavir, and raltegravir on fat and muscle among persons living with HIV. HIV Res Clin Pract. 2020;21(4):91-8.

13. Griesel R, Maartens G, Chirehwa M, Sokhela S, Akpomiemie G, Moorhouse M, et al. CYP2B6 Genotype and Weight Gain Differences Between Dolutegravir and Efavirenz. Clin Infect Dis. 2020.

14. Gorwood J, Ejlalmanesh T, Bourgeois C, Mantecon M, Rose C, Atlan M, et al. SIV Infection and the HIV Proteins Tat and Nef Induce Senescence in Adipose Tissue and Human Adipose Stem Cells, Resulting in Adipocyte Dysfunction. Cells. 2020;9(4).

15. Srinivasa S, Garcia R, Torriani M, Fitch KV, Maehler P, Feldpausch M, et al., editors. UNIQUE MIRNA SIGNATURE IN HIV LIPODYSTROPHY WITH REDUCED ADIPOSE DICER EXPRESSION. Unique miRNA signature in HIV lipodystrophy with reduced adipose Dicer expression CROI 2019 \#683; 2019; Seattle.

16. Srinivasa $S$, Torriani $M$, Fitch KV, Maehler $P$, Iyengar $S$, Feldpausch $M$, et al. Brief Report: Adipogenic Expression of Brown Fat Genes in HIV and HIV-Related Parameters. J Acquir Immune Defic Syndr. 2019;82(5):491-5.

* An very original report on the effect of PI and of HIV infection severity decreasing brown/beige gene expression

17. Olali AZ, Sharma A, Shi $Q$, Hoover DR, Weber KM, French AL, et al. Change in circulating undercarboxylated osteocalcin ( $\mathrm{uCOCN}$ ) is associated with fat accumulation in HIV-seropositive women. J Acquir Immune Defic Syndr. 2020;Publish Ahead of Print.

18. Lake JE, Moser C, Olefsky M, Erlandson KM, Scherzinger A, Stein JH, et al., editors. Changes in fat density after ART initiation CROI 2019 \#681; 2019. 
19. Gelpi M, Ueland PM, Troseid M, Mocroft A, Lebech AM, Ullum H, et al. Abdominal Adipose Tissue Is Associated With Alterations in Tryptophan-Kynurenine Metabolism and Markers of Systemic Inflammation in People With Human Immunodeficiency Virus. J Infect Dis. 2020;221(3):419-27.

* An intersting study showing that products of the tryptophan-kynurenine pathway could be involved in fat-related inflammation

20. Iyengar S, Cote HCF, Fitch KV, Torriani M, Feldpausch M, Srinivasa S. Relationship of Telomere Length to Fat Redistribution in HIV. Open Forum Infect Dis. 2020;7(12):ofaa523.

21. De Vincentis S, Decaroli MC, Fanelli F, Diazzi C, Mezzullo M, Morini F, et al. Health status is related to testosterone, estrone and body fat: moving to functional hypogonadism in adult men with HIV. Eur J Endocrinol. 2021;184(1):107-22.

22. Debroy $\mathrm{P}$, Lake JE, Moser C, Olefsky M, Erlandson KM, Scherzinger A, et al. Antiretroviral therapy initiation is associated with decreased visceral and subcutaneous adipose tissue density in people living with HIV. Clin Infect Dis. 2020.

** A study evaluating the recent marker of AT density which is decreased in ART-initiated PLWH, associated with unhealthy AT and with insulin resistance and dyslipidemia

23. Wanjalla CN, McDonnell WJ, Barnett L, Simmons JD, Furch BD, Lima MC, et al. Adipose Tissue in Persons With HIV Is Enriched for CD4(+) T Effector Memory and T Effector Memory RA(+) Cells, Which Show Higher CD69 Expression and CD57, CX3CR1, GPR56 Coexpression With Increasing Glucose Intolerance. Front Immunol. 2019;10:408.

24. Wanjalla CN, McDonnell WJ, Ram R, Chopra A, Gangula R, Leary S, et al., editors. Increased inflammatory CX3CR1+GPR56+CD57+CD4+ T cells in fat from HIV+ diabetics. CROI $2020 \# 672 ; 2020$; Boston. 
25. Bogorodskaya M, Fitch KV, Lu M, Torriani M, Zanni MV, Looby SE, et al. Measures of Adipose Tissue Redistribution and Atherosclerotic Coronary Plaque in HIV. Obesity. 2020;28(4):749-55.

26. Rubin LH, Gustafson D, Hawkins KL, Zhang L, Jacobson LP, Becker JT, et al. Midlife adiposity predicts cognitive decline in the prospective Multicenter AIDS Cohort Study. Neurology. 2019;93(3):e261-e71.

27. Jankowski CM, Mawhinney S, Wilson MP, Campbell TB, Kohrt WM, Schwartz RS, et al. Body Composition Changes in Response to Moderate- or High-Intensity Exercise Among Older Adults With or Without HIV Infection. J Acquir Immune Defic Syndr. 2020;85(3):340-5. 28. Lagathu C, Bereziat V, Gorwood J, Fellahi S, Bastard JP, Vigouroux C, et al. Metabolic complications affecting adipose tissue, lipid and glucose metabolism associated with HIV antiretroviral treatment. Expert Opin Drug Saf. 2019;18(9):829-40.

29. Lo J, Oyee J, Crawford M, Grove R, DeMasi R, Curtis L, et al., editors. Dolutegravir and Insulin Resistance. CROI \#679; 2019; Seattle.

30. van Wyk J, Ajana F, Bisshop F, De Wit S, Osiyemi O, Portilla Sogorb J, et al. Efficacy and Safety of Switching to Dolutegravir/Lamivudine Fixed-Dose 2-Drug Regimen vs Continuing a Tenofovir Alafenamide-Based 3- or 4-Drug Regimen for Maintenance of Virologic Suppression in Adults Living With Human Immunodeficiency Virus Type 1: Phase 3, Randomized, Noninferiority TANGO Study. Clin Infect Dis. 2020;71(8):1920-9.

31. Assoumou L, Racine C, Fellahi S, Lamaziere A, Farabos D, Beniguel L, et al. Fat gain differs by sex and hormonal status in persons living with suppressed HIV switched to raltegravir/etravirine. AIDS. 2020;34(12):1859-62. 
32. Jeremiah K, Filteau S, Faurholt-Jepsen D, Kitilya B, Kavishe BB, Krogh-Madsen R, et al. Diabetes prevalence by $\mathrm{HbA} 1 \mathrm{c}$ and oral glucose tolerance test among HIV-infected and uninfected Tanzanian adults. PLoS One. 2020;15(4):e0230723.

33. Phuphuakrat A, Nimitphong H, Reutrakul S, Sungkanuparph S. Prediabetes among HIV-infected individuals receiving antiretroviral therapy: prevalence, diagnostic tests, and associated factors. AIDS Res Ther. 2020;17(1):25.

34. Nguyen KA, Peer N, de Villiers A, Mukasa B, Matsha TE, Mills EJ, et al. Glycated haemoglobin threshold for dysglycaemia screening, and application to metabolic syndrome diagnosis in HIV-infected Africans. PLoS One. 2019;14(1):e0211483.

35. Kweka B, Lyimo E, Jeremiah K, Filteau S, Rehman AM, Friis H, et al. Influence of hemoglobinopathies and glucose-6-phosphate dehydrogenase deficiency on diagnosis of diabetes by HbA1c among Tanzanian adults with and without HIV: A cross-sectional study. PLoS One. 2020;15(12):e0244782.

36. Hanttu A, Kauppinen KJ, Kivela P, Ollgren J, Jousilahti P, Liitsola K, et al. Prevalence of obesity and disturbances in glucose homeostasis in HIV-infected subjects and general population - missed diagnoses of diabetes? HIV Med. 2020.

* A study from Finland on recently evaluated PLWH comparing prevalence of prediabetes/diabetes between PLWH and the general population

37. Han WM, Jiamsakul A, Kiertiburanakul S, Ng OT, Sim BL, Sun LP, et al. Diabetes mellitus burden among people living with HIV from the Asia-Pacific region. J Int AIDS Soc. 2019;22(1):e25236.

38. Summers NA, Lahiri CD, Angert CD, Aldredge A, Mehta CC, Ofotokun I, et al. Metabolic Changes Associated With the Use of Integrase Strand Transfer Inhibitors Among Virally Controlled Women. J Acquir Immune Defic Syndr. 2020;85(3):355-62. 
39. Zhang E, Chai JC, Deik AA, Hua S, Sharma A, Schneider MF, et al. Plasma lipidomic profiles and risk of diabetes: two prospective cohorts of HIV-infected and HIV-uninfected individuals. J Clin Endocrinol Metab. 2021.

* A study using a systemic lipidomic approach to identify species associated with diabetes and with potential link with the risk of diabetes.

40. Masters M, Yang J, Lake JE, Abraham A, Kingsley L, Brown TT, et al., editors. Glycemic status and physical function among men with and without HIV CROI 2020 \#683; 2020.

41. Bourgi K, Kundu S, Stewart J, Freiberg M, Gupta S, editors. Greater incidence of diabetes over 10 years among depressed US veterans with HIV CROI 2020; 2020; Boston.

42. Russell E, Albert A, Cote H, Hsieh A, Nesbitt A, Campbell AR, et al. Rate of dyslipidemia higher among women living with HIV: A comparison of metabolic and cardiovascular health in a cohort to study aging in HIV. HIV Med. 2020;21(7):418-28.

43. Sarkar S, Haberlen S, Whelton S, E ES, Kingsley L, Palella F, et al. Greater IL-6, Ddimer, and ICAM-1 Levels Are Associated With Lower Small HDL Particle Concentration in the Multicenter AIDS Cohort Study. Open Forum Infect Dis. 2019;6(12):ofz474.

44. Schwarze-Zander C, Piduhn H, Boesecke C, Schlabe S, Stoffel-Wagner B, Wasmuth JC, et al. Switching tenofovir disoproxil fumarate to tenofovir alafenamide in a real life setting: what are the implications? HIV Med. 2020;21(6):378-85.

45. Lagoutte-Renosi J, Flammang M, Chirouze C, Beck-Wirth G, Bozon F, Brunel AS, et al. Real-Life Impact on Lipid Profile of a Switch from Tenofovir Disoproxil Fumarate to Tenofovir Alafenamide in HIV-Infected Patients. Curr HIV Res. 2021;19(1):84-9.

46. Milinkovic A, Berger F, Arenas-Pinto A, Mauss S, editors. Lipid changes associated with TAF are reversible by switching back to TDF. CROI; 2019; Seattle. 
47. Boccara F, Kumar PN, Caramelli B, Calmy A, Lopez JAG, Bray S, et al. Evolocumab in HIV-Infected Patients With Dyslipidemia: Primary Results of the Randomized, Double-Blind BEIJERINCK Study. J Am Coll Cardiol. 2020;75(20):2570-84.

** An important study showing the efficacy of a PCSK9 inhibitor to lower LDL-C in PLWH with insufficient LDL control.

48. Chai JC, Deik AA, Hua S, Wang T, Hanna DB, Xue X, et al. Association of Lipidomic Profiles With Progression of Carotid Artery Atherosclerosis in HIV Infection. JAMA Cardiol. 2019;4(12):1239-49.

** A large study associating the systemic lipidomic profile with carotid artery atherosclerosis in PLWH 